\title{
Revisiting miR-155 in intervertebral disc degeneration: Blood cell signature and local cell-free profiles
}

\author{
ZHiHENG LIU ${ }^{1,2}$; Zhen SUN ${ }^{3}$; ZHONGYUAN WAN ${ }^{4}$; HaiQIANG WANG ${ }^{2, *}$ \\ ${ }^{1}$ Department of Cardiac Surgery, Xijing Hospital, Air Force Medical University, Xi'an, 710032, China \\ 2 Institute of Integrative Medicine, Shaanxi University of Chinese Medicine, Xi'an, 712046, China \\ ${ }^{3}$ Department of Orthopedics, Xijing Hospital, Air Force Medical University, Xi'an, 710032, China \\ ${ }^{4}$ Department of Orthopedics, Seventh Medical Center of General Hospital of PLA, Beijing, 100700, China
}

Key words: miR-155, PBMCs, Lumbar disc disease, Auto-immunity, Cell death, Exosome

\begin{abstract}
We unraveled the expression profiles of coding-noncoding RNAs in intervertebral disc degeneration (IDD). However, it remains elusive regarding miR-155 expression in peripheral blood mononuclear cells (PBMCs) and local extracellular space in IDD. The study aimed for investigating the miR-155 expression of PBMCs, extracellular miRNAs (ex-miRNAs) of human nucleus pulposus (NP) tissues, and morphological changes of cell death in the IDD process. Here, we harvested peripheral blood and NP samples from scoliosis and IDD patients as control and degenerative groups, respectively. Then standard Ficoll density-gradient centrifugation was used to isolate PBMCs. The two subpopulations of PBMCs were divided based on the cellular difference in adherence, i.e., monocytes/ macrophages and lymphocytes. Quantitative real-time PCR was used to evaluate miR-155 relative expression in PBMCs. ex-miRNAs were screened by comparison between GSE19943, GSE63492, and extracellular RNA (exRNA) atlas. The morphological changes of cell death subtypes in IDD were observed in transmission electron microscopy (TEM). Results indicate that the lower expression of miR-155 in PBMCs from IDD patients ascribed to alterations in monocytes/macrophages. Moreover, we identified 594 shared hsa-miRNAs as the intracellular miRNA expression profile and 258 miRNAs as the ex-miRNA expression profile in human NP tissue. miR-155 was amongst intracellular miRNAs in NP. TEM observation presented multiple endocytic secretory vesicles within human NP cells, implicating exosome transporting machinery. Typical necroptosis and pyroptosis were noted in human NP. Collectively, this study reveals miR-155 expression in PBMCs from IDD patients. Moreover, we propose ex-miRNA expression profile and necroptosis/pyroptosis in human NP tissue, shedding novel light on the etiology of IDD.
\end{abstract}

\section{Introduction}

As the chief cells in nucleus pulposus (NP) tissues, NP cells contribute significantly to the development of intervertebral disc degeneration (IDD), which is the leading cause of low back pain (Richardson et al., 2017). The disc degeneration process depends on the state of NP cells by affecting the extracellular matrix (ECM), intercellular interaction, gene regulation, inflammation, and auto-immunity (Banala et al., 2018). These mechanisms result in the morphologic changes of NP cells and pertaining ECM alterations, consequently leading to disc degeneration in clinical and radiological manifestation. A multitude of studies on NP cells has revealed the underlying mechanisms in IDD for the past

*Address correspondence to: Haiqiang Wang, hqwang@sntcm.edu.cn Received: 16 February 2021; Accepted: 26 March 2021 decades. Specifically, our previous studies illuminated several potential mechanisms for IDD in terms of noncoding RNA (ncRNAs) regulation (Liu et al., 2015), microRNA (miRNAs), long noncoding RNAs (lncRNAs), circular RNAs (circRNAs) (Lan et al., 2016), apoptosis (Wang et al., 2011), immune privilege (Liu et al., 2013; Ma et al., 2015), cytokeratin 8 (CK8) and its phosphorylation (Sun et al., 2013; Sun et al., 2013), Ras association (RalGDS/AF-6) domain family (N-terminal) member 7 (RASSF7) regulation (Liu et al., 2015).

The disc degeneration process goes with cell death (Zhang et al., 2016). With the advancement of detecting techniques and research methodology, the concept and classification of cell death have been updated greatly. Regulated necrosis was defined as apoptosis in 1972 (Kerr et al., 1972). So far, regulated active necrosis has expanded to a number of forms, including necroptosis (characterized by the necrosome consisting of receptor-interacting protein 
1 (RIPK1), receptor-interacting protein 3 (RIPK3), and mixed lineage kinase domain-like protein (MLKL), pyroptosis (poreforming protein GSDMD as effector), and ferroptosis (Green, 2019). Moreover, about 12 subtypes of cell death have been established by the Nomenclature Committee on Cell Death in 2018 (Galluzzi et al., 2018).

Although abundant studies on NP cell death are available in the literature, the mechanisms have not been clarified to a large extent (Zhang et al., 2016). Further investigation needs a holistic view of the poly-etiological theory in IDD (Hemanta et al., 2016). Our previous studies on gene regulation in IDD established GEO SuperSeries Datasets: GSE67567 (Lan et al., 2016), comprising miRNA profiles (GSE19943 (Wang et al., 2011) with RNA from fresh isolated NP cells, GSE63492 (Liu et al., 2015; Lan et al., 2016) with total RNA from NP tissues), mRNA and lncRNA profiles (GSE56081 (Wan et al., 2014)), circRNA profile (GSE67566 (Lan et al., 2016)). Amongst the differential expressed miRNAs, we revealed the regulatory effect of miR155 in NP cell apoptosis (Wang et al., 2011). Our further study of IDD in NP cell morphology changes (cell clusters), immune privilege (Liu et al., 2013), and lncRNAs shed light on the auto-immunity etiology of IDD.

The main components of human intervertebral discs are central gel-like nucleus pulposus, surrounding annulus fibrosus, and adjacent endplates. Lumbar disc degeneration (LDD) can be graded in terms of multiple resolutions. We summarize the 6 grading systems, including diagnostic imaging as magnetic resonance imaging as we noted (Kerr et al., 1972; Zhang et al., 2016), gross morphology (Green, 2019), histological changes (Galluzzi et al., 2018), ultrastructural alterations (Hemanta et al., 2016), gene deregulation (Wang et al., 2011; Wan et al., 2014; Liu et al., 2015; Hemanta et al., 2016) and RNA profiles (Livak and Schmittgen, 2001; Murillo et al., 2019).

Recently, exRNA Atlas presents the first line of exRNA resource (https://exrna-atlas.org) by the NIH Extracellular
RNA Communication Consortium (Murillo et al., 2019). The cutting-edge Atlas version $4 \mathrm{P} 1$ covers 5 human biofluids, including serum, plasma, cerebrospinal fluid, saliva, and urine, merging exRNA experimental data from 19 available studies. However, there have been no lines of evidence revealing exRNA profiles in the extracellular space of solid organs or tissues. This is an important omission owing to the solid hallmarks of the main vital organs throughout the body.

In light of the interaction between NP and systemic immune cells, the aim of the present study is twofold: (1) to unravel the role of miR-155 of PBMCs in the development of IDD and (2) to unravel ex-miRNAs in human NP tissues.

\section{Materials and Methods}

\section{Sample collection}

Because of the difficulties in obtaining NP tissues from healthy individuals, NP tissues in this study were collected from IDD and idiopathic scoliosis patients as degenerative and control samples, respectively. Four cases of scoliosis NP tissues and 8 cases of LDD NP tissues were collected in this study. Patients' demographic data were shown in Tab. 1. Obtained specimens were classified by MRI scans followed Pfirrmann and colleagues' classification system (Pfirrmann et al., 2001), in which idiopathic scoliosis discs and IDD discs were considered as Grade II and Grade IV, respectively. NP cells were isolated and cultured from discectomy acquired NP tissues followed our previous method (Wang et al., 2011; Liu et al., 2013). Passages 1 or 2 of cultured NP cells were observed by microscope first.

\section{TEM}

Human NP tissues and digested NP cells were first fixed with a mixture of $2 \%$ paraformaldehyde and $2 \%$ glutaraldehyde with phosphate buffer at $\mathrm{pH} 7.4$, then postfixed with a $1 \%$ solution

TABLE 1

Demographic hallmarks of participating patients

\begin{tabular}{lllll}
\hline Sample & Age (years) & Gender & BioSample ID & Group \\
\hline 1 & 18 & Female & GSM498350 & $\begin{array}{l}\text { Idiopathic scoliosis } \\
\text { T5/6-T11/12 } \\
\end{array}$ \\
2 & 20 & Female & GSM498351 & $\begin{array}{l}\text { Idiopathic scoliosis } \\
\text { T5/6-T10/11 }\end{array}$ \\
3 & 30 & Female & GSM498352 & Idiopathic scoliosis \\
4 & 11 & Female & - & Idiopathic scoliosis \\
5 & 33 & Female & GSM498355 & LDD: L5/S1 \\
6 & 59 & Female & - & LDD: L3-L5 \\
7 & 70 & Female & - & LDD: L1/2 \\
8 & 30 & Male & - & LDD: L4/5 \\
9 & 73 & Female & - & LDD: L3-5 \\
10 & 74 & Male & - & LDD: L3-S1 \\
11 & 46 & Male & - & LDD: L4/5 \\
12 & 66 & Female & - & LDD: L5/S1 \\
\hline
\end{tabular}

Note: LDD: lumbar disc disease. BioSample numbers: registered in NCBI BioSample database for microarray studies using nucleus pulposus tissues. 
of osmium tetroxide which contains $1.5 \%$ potassium ferrocyanide. After three times washes in phosphate, graded alcohols were used to dehydrating, and Epon was used to embed the samples. Ultrathin sections were stained by uranyl acetate and lead citrate for $15 \mathrm{~min}$, respectively, and observed in TEM of Model JEM 2000 EX (Japan Electron Organization, Tokyo, Japan) under an accelerating voltage of $80 \mathrm{kV}$.

\section{PBMCs collection and subpopulation division}

PBMCs were isolated from blood samples of patients with idiopathic scoliosis, and IDD followed standard Ficoll density-gradient centrifugation. The two subpopulations of PBMCs, i.e., monocytes/macrophages and lymphocytes, were divided based on the cellular difference in adherence as previously described.

\section{RNA isolation}

The total RNA of NP tissues and isolated cells was extracted by TRIzol (Invitrogen) and RNeasy mini kit (QIAGEN). Electrophoreses in $8 \%$ denaturing polyacrylamide gels were used to monitor the integrity of RNA.

\section{RT-PCR}

TaqMan miRNA assays were used to detected miR-155 expression in PBMCs. High-Capacity cDNA Archive Kit (ABI) was used to reverse transcribe to cDNAs. RNA concentrations were detected by a NanoDrop instrument (NanoDrop Technologies). U6 controls were used to normalize the levels of miR-155. All RT reactions were run in GeneAmp PCR 9700 Thermocycler (Applied Biosystems). The relative expression of miR-155 was evaluated following the comparative $\mathrm{Ct}(2-\Delta \Delta \mathrm{Ct})$ method (Livak and Schmittgen, 2001).

\section{Ex-miRNAs analysis}

We used Venny 2.1.0 (Juan Carlos Oliveros) for a profound analysis of microarray miRNA expression profiles, distinguishing differential miRNAs in terms of intra- or extra-cellular location.

\section{Statistics}

The comparison of miR-155 expression between monocytes/ macrophages and lymphocytes, the comparison of miR-155 expression in IDD and control patients were all performed by Student's $t$-test. $P<0.05$ is considered statistically significant.

\section{Results}

The lower expression of miR-155 in PBMCs from patients with IDD

RT-PCR found that lower expression of miR-155 in PBMCs from IDD patients $(0.23$-fold, Fig. 1A). Given that PBMCs comprise two main subpopulations of cells (i.e., monocytes/ macrophages and lymphocytes), we further compared miR155 expression between monocytes/macrophages and lymphocytes based on the cellular difference in adherence between the two subpopulations (Pauley et al., 2008). We found that miR-155 was expressed at a similar level in lymphocytes as in monocytes/macrophages in control patients (Fig. 1B, $P>0.05$ ), whereas higher expression of miR-155 was in lymphocytes than in monocytes/macrophages in IDD patients (Fig. $1 \mathrm{C}, P<0.05$ ). Therefore, the lower expression of miR-155 in PBMCs was ascribed to the alterations of miR-155 expression in monocytes/macrophages.

Ex-miRNA profile in human spinal disc: expanding exRNA atlas MiRNA expression in human NP was analyzed from GSE19943 (miRCURY Version 11.0, total RNA was isolated from fresh NP cell suspension of NP tissues (Wang et al., 2011)) and GSE63492 (miRCURY Version 18.0, total RNA was extracted from NP tissues by TRIspin methods (Livak and Schmittgen, 2001; Lan et al., 2016)). Since microarray probes were based on platforms from Exiqon with minimal inter-platform bias, we compared the 2 miRNA profiles for detecting of ex-miRNAs in human lumbar disc tissue (Tab. 2).

In total, there were 1278 hsa-miRNAs captured for GSE19943 and 1916 hsa-miRNAs for GSE63492. Comparing miRNA expression profiles of GSE19943 and
A

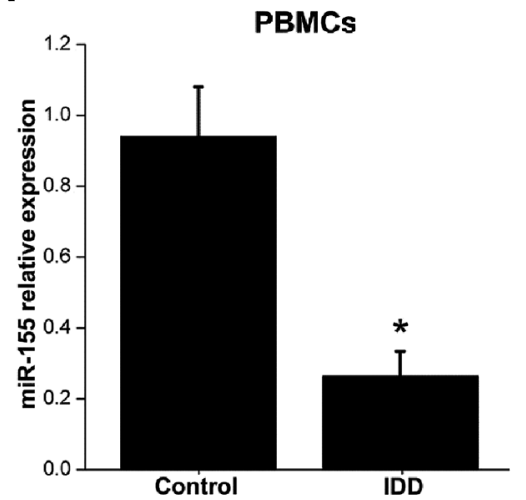

B

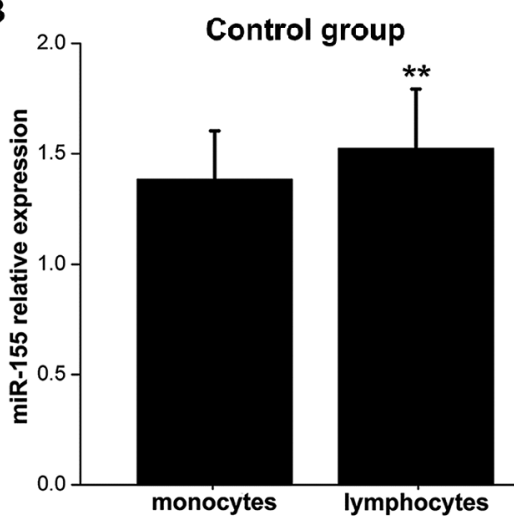

C

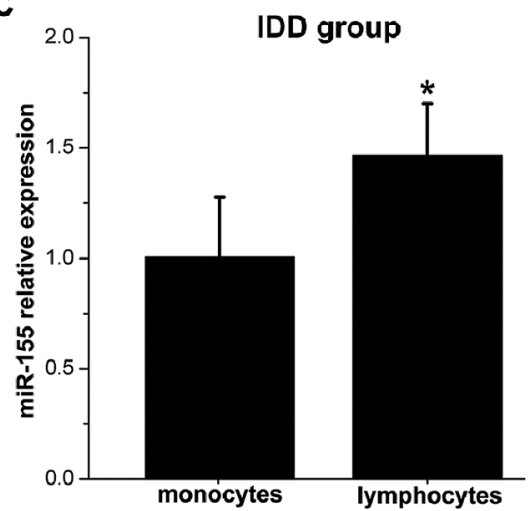

FIGURE 1. Lower expression of miR-155 in PBMCs from the IDD group and the alterations of miR-155 expression in lymphocytes mainly contribute to the downregulation of miR-155. (A) lower expression of miR-155 in PBMCs (0.23-fold). The expression of miR-155 in PBMCs from control and IDD patients was measured by qRT-PCR using TaqMan miRNA assays. The relative amounts of miR-155 were evaluated following the comparative $\mathrm{Ct}(2-\Delta \Delta \mathrm{Ct})$ method. The mean $\pm \mathrm{SEM}$ is shown. ${ }^{\star} P<0.05$. (B) miR-155 expression is almost at the same level in the lymphocytes subgroup as in the monocytes/macrophages subgroup. The two subgroups were isolated according to their difference in adherence. The relative amounts of miR-155 in each subgroup were evaluated as aforementioned. ${ }^{\star \star} P>0.05$. (C) miR-155 expression is at a higher level in the lymphocytes subgroup than in the monocytes/macrophages subgroup $(1.25$-fold $) .{ }^{\star} P<0.05$. All experiments were repeated at least three times. 
TABLE 2

miRNA expression profiles in GSE19943 and GSE63492

\begin{tabular}{|c|c|c|c|c|}
\hline Accession number & Control/Disease & Hsa-miRNA profile & Shared has-miRNAs & Specific has-miRNAs \\
\hline GSE19943 & $\begin{array}{l}\text { Scoliosis control: } \\
\text { GSM498350 } \\
\text { GSM498351 } \\
\text { GSM498352 } \\
\text { LDD: } \\
\text { GSM498353 } \\
\text { GSM498354 } \\
\text { GSM498356 }\end{array}$ & 1278 & 594 & 667 \\
\hline GSE63492 & $\begin{array}{l}\text { Normal: } \\
\text { GSM1551024 } \\
\text { GSM1551025 } \\
\text { GSM1551026 } \\
\text { GSM1551027 } \\
\text { GSM1551028 } \\
\text { LDD: } \\
\text { GSM1551029 } \\
\text { GSM1551030 } \\
\text { GSM1551031 } \\
\text { GSM1551032 } \\
\text { GSM1551033 }\end{array}$ & 1916 & & 1295 \\
\hline
\end{tabular}

GSE63492, we identified 594 shared hsa-miRNAs as intracellular miRNA expression profiles. Notably, there were 667 specific miRNAs in GSE19943, termed as the scoliosisspecific miRNA expression profile. There were 1295 specific hsa-miRNAs in GSE63492; further comparison with the exRNA atlas, which contains human plasma miRNA profile of 669 miRNAs, we identified 258 shared miRNAs as the ex-miRNA expression profile in human NP tissue of lumbar discs (Fig. 2). MiR-155 was in NP intracellular profile.

Necroptosis/pyroptosis features and exosomes under TEM

Cell cluster formation as histological hallmarks of IDD was noted before TEM observation (Fig. 3A). Plasma membrane rupture, cell swelling, and lysis were noted as both the

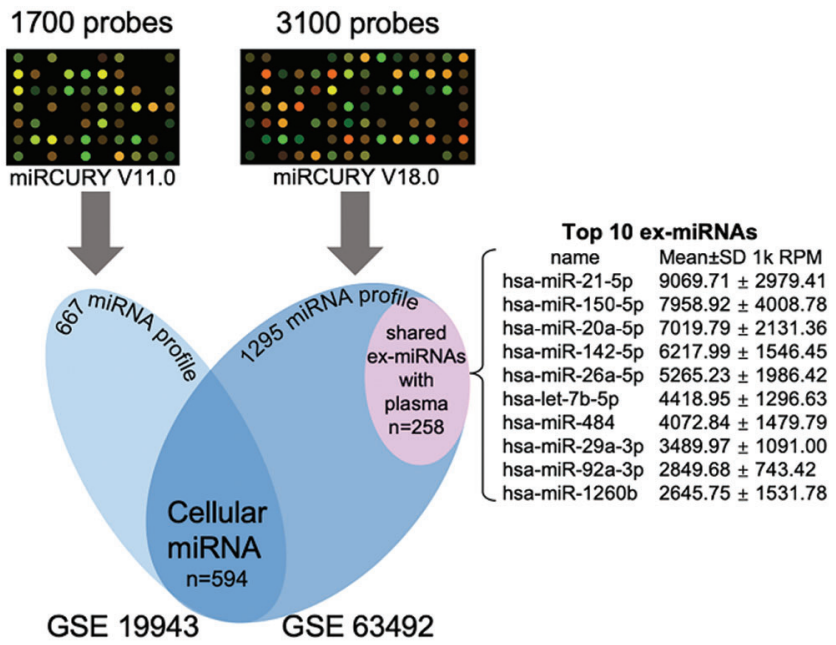

FIGURE 2. Data analysis diagram indicating ex-miRNA expression profile of human NP tissue. necroptosis and pyroptosis characteristics (Fig. 3B). Inflammasome/pyroptosome-induced membrane rupture was observed as pyroptosis morphological features (Fig. 3C). Vacuolization in mitochondria was noted as an early stage of mitochondrial membrane permeability transitiondependent (MPT-dependent) necrosis (Fig. 3D).

\section{Discussion}

Based on one decade's studying accumulation, we are the first to present the downregulation of miR-155 in PBMCs from LDD patients, implicating the immune interaction between PBMCs and NP cells in LDD via miR-155. Moreover, necroptosis and pyroptosis features of NP cells are presented with TEM evidence for the first time. ex-miRNA expression profiles are putatively proposed, expanding human body fluid exRNA atlas. In fact, there are studying groups reporting apoptosis and necrosis in intervertebral disc (Zhao et al., 2007; Velikonja et al., 2014). The key-cell NP cells have always been the research focus in IDD. Our previous results demonstrated that the degenerative NP may still preserve the capability of maintaining homeostasis of the cell population (Chen et al., 2013). The state of NP cells at the micro-level determines disc-related diseases to some extent. With the constantly updated conception of cell death (Galluzzi et al., 2018), the subtypes of apoptosis in IDD need further exploration. Notably, the mechanisms underlying necroptosis or pyroptosis in IDD have been scant in the literature. Our study filled the void by providing TEM evidence, whereas underlying mechanisms of necroptosis and pyroptosis need further studies.

Intercellular interactions determined the state and fate of the cell (Velikonja et al., 2014), impact the ECM 

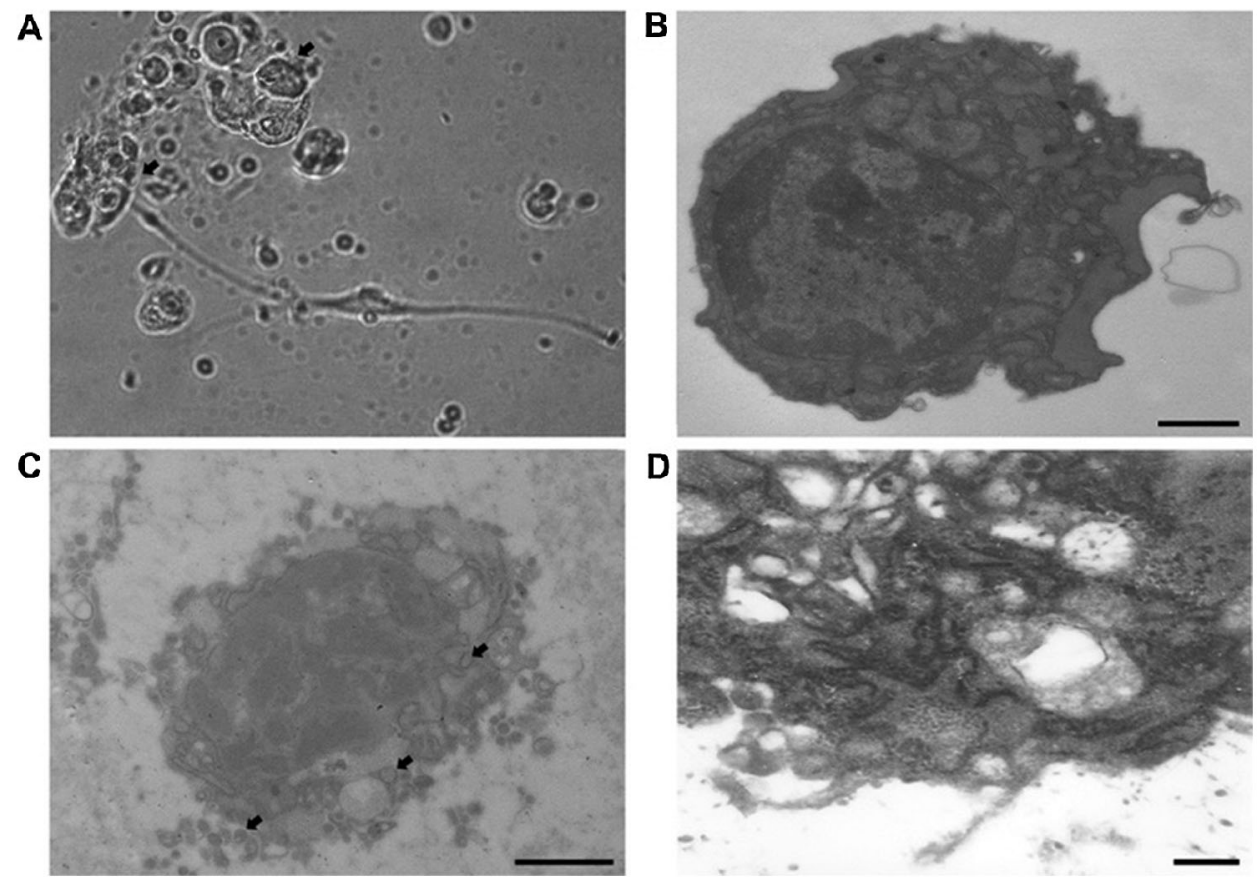

FIGURE 3. Representative photographs of human fresh isolated NP cell suspension (A) and TEM section (B, C, D). (A). Note the cell cluster formation as histological hallmarks of intervertebral disc degeneration (Arrow, original magnification 400×). (B) Typical plasma membrane rupture, cell swelling, and lysis were noted as both the necroptosis and pyroptosis characteristics under electron microscopy. Scale bar $=1 \mu \mathrm{m}$. (C) Inflammasome/pyroptosome-induced membrane rupture was observed as pyroptosis morphological features under electron microscopy. The diameter of the vesicles (Arrow) is about $30-100 \mathrm{~nm}$. Scale bar $=1 \mu \mathrm{m}$. (D) Note the vacuolization in mitochondria under electron microscopy, early stage of MPT necrosis. Scale bar $=100 \mathrm{~nm}$.

(Feng et al., 2006) and organ's function via diverse pathways. MicroRNAs (miRNAs) were one of the important signal transmitters in numerous biologic processes (Li et al., 2015).

MiRNAs, an average of 22 nucleotides small RNAs were involved in the regulation of cell differentiation, proliferation, and apoptosis has been better understood. Our comparison of miRNA expression profiles between GSE19943 and GSE63492 screened out 594 shared hsa-miRNAs as intracellular miRNA expression profile, and miR-155 was amongst the profile. Our previous study (Wang et al., 2011) showed lower expression of miR-155 in human degenerative NP, which localizes in the cytoplasm of human NP cells, which can regulate apoptosis of NP cells through inhibiting FADD and CASP3. For the multiple regulatory roles in neoplasm, immune response (Luo et al., 2013), inflammation (Ochs et al., 2011), apoptosis (Lima et al., 2011), and virus infection, the role of miR-155 in autoimmunity and intercellular interaction of IDD has not been well addressed. In this study, we affirmed lower expression of miR-155 in PBMCs from patients with IDD, which is mainly manifested in monocytes/macrophages rather than in lymphocytes.

The maintenance of the immune privilege of certain organs results from the interaction between immune and tissue cells via various pathways other than the physiology barrier of blood supply (Sun et al., 2013; Ma et al., 2015). The avascular structure of the intervertebral disc means the autoimmunity plays an important role in disc function maintenance. PBMCs may be the vital cells in disc autoimmunity. PBMCs are mainly composed of monocytes/macrophages and lymphocytes, which make up the defense system at the cellular level. PBMCs can initiate a series of immune responses when NP tissues ruptured from AF (Liu et al., 2013).
It is well established that both Fas ligand (FasL) and Fas are expressed in human Cytotoxic T lymphocytes (CTLs) ( $\mathrm{He}$ and Ostergaard, 2007) and NP cells, and both FasL-induced and mitochondrial-dependent pathways exist in human NP cells. Previous studies have found that the expression of FasL decreases (Kaneyama et al., 2008), and the expression of Fas increases (Sun et al., 2013) in the disc degeneration process due to unknown reasons, which might attenuate the immune privilege of NP and result in increased cell death. Furthermore, the downregulation of miR-155 in NP seems to raise the susceptibility of NP cells to death (Wang et al., 2011). Therefore, the downregulation of miR-155 in PBMCs might regulate the network of Fas/FasL between NP cells and CTLs and increase the death susceptibility of NP cells and CTLs. In this study, down-regulation of miR-155 in IDD patients ascribe to monocyte/macrophages partly, regulating disc autoimmunity via Fas-FasL apoptosis pathways.

Monocytes/macrophages from PBMCs are also an important part of autoimmunity. The morphologic evidence from our previous studies showed multiple potentials of NP cells, building and sweeping ECM and nonviable NP cells as phagocytic cells (Chen et al., 2013). And phagocytic NP cells displayed morphologic hallmarks of both NP cells and macrophages, which indicates that those cells belong to histiocytes. Furthermore, in some parts of the human body, macrophages could fuse to form multi-nuclear giant cells after phagocytosis, i.e., Langerhans cells. Our findings indicate that monocytes/macrophages from periphery blood regulate disc autoimmunity by interacting with NP cells in the process of LDD via miR-155. Besides the regulatory effect in apoptosis, miR-155 might trigger downstream 
immune response via the caspase-8-NLRP3 pathway, which mainly exists in macrophages (Feltham et al., 2017). This study identified the inflammasome/pyroptosome of NP cells under the TEM sections, and we screened out ex-miRNAs of NP tissue and intracellular miRNAs of NP cells. Further study needs to unravel the interaction between NP cells and immunocytes in necroptosis/pyroptosis of disc autoimmunity.

This study represents the immune interaction between PBMCs and NP cells in LDD by combining with our previous series of investigations. Human IDD can trigger a systemic reaction with the involvement of PBMCs, and miR-155 can modulate the process of IDD as well as the autoimmunity of human NP. Further studies are needed to clarify the precise mechanisms between PBMCs and NP cells, such as exosomes, pyroptosis, and the inflammasome.

Acknowledgement: We thank KangChen Bio-tech Inc. (Shanghai, China) for their technical support on qRT-PCR. HQW conceived this research and carried out TEM observation; ZHL, ZS, and ZYW collected NP samples, prepared tissues and cells, and analyzed microarray miRNA expression profiles. All authors prepared the manuscript and approved the final content.

Author Contribution: The authors confirm contribution to the paper as follows: study conception and design: HQW, ZHL; data collection: ZHL, ZS; analysis and interpretation of results: ZHL, ZS, ZYW; draft manuscript preparation: ZHL, HQW. All authors reviewed the results and approved the final version of the manuscript.

Availability of Data and Materials: The processed raw data of ex-miRNAs were uploaded in Mendeley Datasets (http:// dx.doi.org/10.17632/42khxv38hf.1).

Ethics Approval and Consent to Participate: Ethical approval of our series studies was attained by the Ethics Review Board of Xijing Hospital, Fourth Medical Military University (Approval Nos. 20090611-3, 20111103-7, KY20150312-4) in terms of experimental application of human surgical NP tissues and collecting peripheral blood samples from patients. Written informed consent was obtained for each participant or his/ her relatives.

Funding Statement: This study was supported by the National Natural Science Foundation of China (Grant No. 81572182).

Conflicts of Interest: The authors have no conflict of interest to disclose.

\section{References}

Banala RR, Sampara P, Vemuri SK, Gurava Reddy AV, Gpv S (2018). Understanding the molecular biology of intervertebral disc degeneration and potential gene therapy strategies for regeneration: A review. Gene Therapy 25: 67-82. DOI 10.1038/s41434-018-0004-0.

Chen YF, Zhang YZ, Zhang WL, Luan GN, Liu ZH et al. (2013). Insights into the hallmarks of human nucleus pulposus cells with particular reference to cell viability, phagocytic potential and long process formation. International Journal of Medical Sciences 10: 1805-1816. DOI 10.7150/ijms.6530.
Feltham R, Vince JE, Lawlor KE (2017). Caspase-8: Not so silently deadly. Clinical \& Translational Immunology 6: e124. DOI 10.1038/cti.2016.83.

Feng H, Danfelter M, Strömqvist B, Heinegård D (2006). Extracellular matrix in disc degeneration. JBJS 88: 25-29.

Galluzzi L, Vitale I, Aaronson SA, Abrams JM, Adam D et al. (2018). Molecular mechanisms of cell death: Recommendations of the Nomenclature Committee on Cell Death 2018. Cell Death \& Differentiation 25: 486-541. DOI 10.1038/s41418017-0012-4.

Green DR (2019). The coming decade of cell death research: Five riddles. Cell 177: 1094-1107. DOI 10.1016/j.cell.2019.04.024.

He JS, Ostergaard HL (2007). CTLs contain and use intracellular stores of FasL distinct from cytolytic granules. Journal of Immunology 179: 2339-2348. DOI 10.4049/jimmunol.179.4.2339.

Hemanta D, Jiang XX, Feng ZZ, Chen ZX, Cao YW (2016). Etiology for degenerative disc disease. Chinese Medical Sciences Journal 31: 185-191. DOI 10.1016/S1001-9294(16)30049-9.

Kaneyama S, Nishida K, Takada T, Suzuki T, Shimomura T et al. (2008). Fas ligand expression on human nucleus pulposus cells decreases with disc degeneration processes. Journal of Orthopaedic Science 13: 130-135. DOI 10.1007/s00776-0071204-4.

Kerr JF, Wyllie AH, Currie AR (1972). Apoptosis: A basic biological phenomenon with wide-ranging implications in tissue kinetics. British Journal of Cancer 26: 239-257. DOI 10.1038/bjc.1972.33.

Lan PH, Liu ZH, Pei YJ, Wu ZG, Yu Y et al. (2016). Landscape of RNAs in human lumbar disc degeneration. Oncotarget 7: 63166-63176. DOI 10.18632/oncotarget.11334.

Li Z, Yu X, Shen J, Chan MT, Wu WKK (2015). MicroRNA in intervertebral disc degeneration. Cell Proliferation 48: 278283. DOI 10.1111/cpr.12180.

Lima RT, Busacca S, Almeida GM, Gaudino G, Fennell DA, Vasconcelos MH (2011). MicroRNA regulation of core apoptosis pathways in cancer. European Journal of Cancer 47: 163-174. DOI 10.1016/j.ejca.2010.11.005.

Liu X, Che L, Xie YK, Hu QJ, Ma CJ et al. (2015). Noncoding RNAs in human intervertebral disc degeneration: An integrated microarray study. Genomics Data 5: $80-81$. DOI 10.1016/j. gdata.2015.05.027.

Liu ZH, Huo JL, Wu ZG, Sun Z, Bai F et al. (2015). RASSF7 expression and its regulatory roles on apoptosis in human intervertebral disc degeneration. International Journal of Clinical and Experimental Pathology 8: 16097-16103.

Liu ZH, Sun Z, Wang HQ, Ge J, Jiang TS (2013). FasL expression on human nucleus pulposus cells contributes to the immune privilege of intervertebral disc by interacting with immunocytes. International Journal of Medical Sciences 10: 1053-1060. DOI 10.7150/ijms.6223.

Livak KJ, Schmittgen TD (2001). Analysis of relative gene expression data using real-time quantitative PCR and the $2-\Delta \Delta \mathrm{Ct}$ Method. Methods 25: 402-408. DOI 10.1006/meth.2001.1262.

Luo X, Ranade K, Talker R, Jallal B, Shen N, Yao Y (2013). microRNA-mediated regulation of innate immune response in rheumatic diseases. Arthritis Research \& Therapy 15: 210. DOI 10.1186/ar4194.

Ma C, Liu X, Che L, Liu Z, Samartzis D, Wang H (2015). Stem cell therapies for intervertebral disc degeneration: Immune privilege reinforcement by Fas/FasL regulating machinery. Current Stem Cell Research \& Therapy 10: 285-295. DOI 10.2174/1574888X10666150416114027. 
Murillo OD, Thistlethwaite W, Rozowsky J, Subramanian SL, Lucero R et al. (2019). exRNA atlas analysis reveals distinct extracellular RNA cargo types and their carriers present across human biofluids. Cell 177: 463-477. DOI 10.1016/j.cell.2019.02.018.

Ochs MJ, Steinhilber D, Suess B (2011). MicroRNA involved in inflammation: Control of eicosanoid pathway. Frontiers in Pharmacology 2: 39. DOI 10.3389/fphar.2011.00039.

Pauley KM, Satoh M, Chan AL, Bubb MR, Reeves WH, Chan EK (2008). Upregulated miR-146a expression in peripheral blood mononuclear cells from rheumatoid arthritis patients. Arthritis Research \& Therapy 10: R101. DOI 10.1186/ar2493.

Pfirrmann CW, Metzdorf A, Zanetti M, Hodler J, Boos N (2001). Magnetic resonance classification of lumbar intervertebral disc degeneration. Spine 26: 1873-1878. DOI 10.1097/ 00007632-200109010-00011.

Richardson SM, Ludwinski FE, Gnanalingham KK, Atkinson RA, Freemont AJ, Hoyland JA (2017). Notochordal and nucleus pulposus marker expression is maintained by subpopulations of adult human nucleus pulposus cells through aging and degeneration. Scientific Reports 7: 1356. DOI 10.1038/s41598-017-01567-w.

Sun Z, Guo YS, Yan SJ, Wan ZY, Gao B et al. (2013). CK8 phosphorylation induced by compressive loads underlies the downregulation of CK8 in human disc degeneration by activating protein kinase C. Laboratory Investigation 93: 1323-1330. DOI 10.1038/labinvest.2013.122.

Sun Z, Wan ZY, Liu ZH, Guo YS, Yin JB et al. (2013). Expression of soluble Fas and soluble FasL in human nucleus pulposus cells.
International Journal of Clinical and Experimental Pathology 6: 1567-1573.

Sun Z, Wang HQ, Liu ZH, Chang L, Chen YF et al. (2013). Downregulated $\mathrm{CK} 8$ expression in human intervertebral disc degeneration. International Journal of Medical Sciences 10: 948-956. DOI 10.7150/ijms.5642.

Sun Z, Zhang M, Zhao XH, Liu ZH, Gao Y et al. (2013). Immune cascades in human intervertebral disc: The pros and cons. International Journal of Clinical and Experimental Pathology 6: 1009-1014.

Velikonja NK, Urban J, Fröhlich M, Neidlinger-Wilke C, Kletsas D et al. (2014). Cell sources for nucleus pulposus regeneration. European Spine Journal 23: 364-374. DOI 10.1007/s00586-013-3106-9.

Wan ZY, Song F, Sun Z, Chen YF, Zhang WL et al. (2014). Aberrantly expressed long noncoding RNAs in human intervertebral disc degeneration: A microarray related study. Arthritis Research \& Therapy 16: 2163. DOI 10.1186/s13075-014-0465-5.

Wang H, Yu X, Liu Z, Cheng X, Samartzis D et al. (2011). Deregulated miR-155 promotes Fas-mediated apoptosis in human intervertebral disc degeneration by targeting FADD and caspase-3. Journal of Pathology 225: 232-242. DOI 10.1002/path.2931.

Zhang F, Zhao X, Shen H, Zhang C (2016). Molecular mechanisms of cell death in intervertebral disc degeneration (Review). International Journal of Molecular Medicine 37: 1439-1448. DOI 10.3892/ijmm.2016.2573.

Zhao CQ, Wang LM, Jiang LS, Dai LY (2007). The cell biology of intervertebral disc aging and degeneration. Ageing Research Reviews 6: 247-261. DOI 10.1016/j.arr.2007.08.001. 